\title{
Experience Report: Association Between Flow Chart, Electronic Patient Record and Telephone Monitoring in the Success of Fighting Dengue Feaver in the Hospital and Emergency Services in São Bernardo do Campo, Brazil
}

\author{
Andrea M. Losacco*, Eliana V. Miranda, Renata Martello, Karla Possendoro, Meire A. \\ Pinheiro and Gabriela Falchi
}

Hospital e Pronto Socorro Central, São Bernardo do Campo, Brazil

\section{Objective}

Report successful experience in fighting dengue fever in the Hospital and Emergency Services in São Bernardo do Campo, joining the flowchart included, telephone monitoring and Electronic Patient Records.

\section{Introduction}

Dengue fever is a dynamic infectious disease, allowing the patient to rapidly move from one stage to another during its course. Proper management of patients depends on early recognition of warning signs, continuous monitoring and re-staging cases and prompt fluid replacement. The telemedicine and Electronic Patient Records (EPR) belong to a series of advances of new features such as decision-making support systems including efforts on health monitoring, in view of the EPR as a support tool to allow the association of welfare activities as a database for the management of epidemiological information and monitoring. In addition, telemonitoring systems can be used for the monitoring of patients with chronic diseases in their homes which leads to cost savings in hospitalization and ensures appropriate care and the proper development of these patients. The continuous remote monitoring of these patients decreases the amount of hospital visits for monitoring procedures, also facilitating successful treatment, as in the fever dengue cases.

\section{Methods}

This study, descriptive in character, was conducted at the Hospital and Emergency Services, in the municipality of São Bernardo do Campo, São Paulo, which deals with coronary emergency cases, trauma, infectious and chronic diseases with prolonged hospitalization; composed of 150 beds, 10 beds for general adult ICU and 05 beds for pediatric ICU. Suspected cases of dengue fever treated in the months of January to May 2015 formed the study population. Validation, training and implementation of a screening flowchart of suspected dengue fever cases treated at hospital was performed using a patient identification form and collection of signs and symptoms including the tourniquet test, related to the disease. For Tourniquet Test training, posters were made describing the proper technique for performing the test, and the QR Code* of the educational video on the technique was placed on the poster so that each employee could view the instructional video on their own smartphone. There was a daily tracking of all records in the EPR, tracking the medical and nursing history and laboratory results, and the records selected that contained in their records the words Dengue and/or the words Fever, Retro-orbital Pain, Headache, Myalgia or Arthralgia, recording 1,298 cases; of which 38 had signs and symptoms of severity and required hospitalization, progressing satisfactorily. For each selected event, phone monitoring was conducted, with information on the progress of the case, timely collection of general serology and guidelines such as the need for adequate hydration during the course of the disease, in addition to warning patients for them to return to the emergency room if they exhibit signs of worsening.

\section{Results}

1,298 patients were attended with suspected dengue fever. The main symptoms exhibited were fever in $99 \%$ of cases, accompanied by mialgia in $89 \%$ of cases, headache in $82 \%$ and retro-orbital pain in $71 \%$. Symptoms more closely related to severe forms were vomiting, and bleeding. The prevalence of positivity was $41 \%$ which can be low when compared to other services, but the lack of timely collection of the serology may have inhibited this data. Comparing the patients with positive serology with those with negative serology, but who developed a feverish state similar to dengue, it was found that the symptoms of retro-orbital pain, itching and rash exhibited statistically significant differences in dengue fever cases.

\section{Conclusions}

The monitoring to guide appropriate measures regarding the disease were decisive, we managed to notify and $100 \%$ of the cases in a timely manner, thus avoiding complications and keeping the deaths at zero. The EPR use in order to improve the quality of the epidemiological indicators produced as part of the local surveillance of all cases of diseases of compulsory notification, is essential.

\section{Keywords}

Dengue Fever; Data Record Patient; Surveillance

\section{References}

1. TDR/WHO. Dengue: Guidelines for Diagnosis, Treatment, Prevention and Control(TDR/WHO, Geneva, Switzerland, 2009).

2. Klompas, Michael, et al. "Automated identification of acute hepatitis B using electronic medical record data to facilitate public health surveillance." PLoS One 3.7 (2008).

\section{*Andrea M. Losacco}

E-mail: a.losacco@gmail.com 